\title{
Políticas de género de ámbito global en el control y la prevención del tabaquismo
}

\author{
Isabel Nerín, PhD, (I) Mireia Jané, PhD. ${ }^{(2)}$
}

\begin{abstract}
Nerín I, Jané M.
Políticas de género de ámbito global en el control y la prevención del tabaquismo. Salud Publica Mex 2010;52 supl 2:S304-S3I4.
\end{abstract}

\begin{abstract}
Resumen
Las fases más avanzadas de la epidemia tabáquica muestran que las desigualdades sociales, en general, y las de género, en particular, favorecen el consumo de tabaco entre las mujeres y los grupos sociales más desfavorecidos. Dada la creciente prevalencia de tabaquismo entre las mujeres, resulta prioritario incorporar la perspectiva de género en el diseño de políticas de prevención y tratamiento del tabaquismo. Tras breve mención del marco global del control del tabaquismo, se describen las diferentes políticas con las estrategias mínimas que deberían incluir los aspectos específicos de género. Entre ellas se incluyen medidas de empoderamiento destinadas, en principio, a disminuir la desigualdad de género; medidas legislativas; medidas asistenciales $y$, por último, medidas relacionadas con la investigación y la evaluación, con el objetivo de incorporar la perspectiva de género tanto en la elaboración de políticas como en el análisis del problema.
\end{abstract}

Palabras clave: género; tabaco; políticas
Nerín I, Jané M.

Global gender policies

in prevention and tobacco control.

Salud Publica Mex 2010;52 suppl 2:S304-S3 I4.

\section{Abstract}

Later stages of the smoking epidemic show that social and gender inequalities increase tobacco use among women and more deprived social groups. Given that smoking prevalence among women is increasing adding gender perspective in the design of prevention and tobacco control policies is a priority. After a brief description of the overall frame of tobacco control, different policies including minimum strategies that should incorporate gender specific aspects are described. Among them, empowerment measures addressed to reduce gender inequities are included; legislative measures; health care measures; and lastly measures related to research and evaluation aiming to add gender perspective in tobacco policy implementation and analysis.

Key words: gender; tobacco; policies
A ctualmente se considera el tabaquismo como una adicción, debido a la capacidad adictiva de la nicotina. Al igual que en otras adicciones, el consumo de tabaco está determinado no sólo por las características de la sustancia, sino por factores del individuo y del entorno.
Hace más de 10 años, López y colaboradores ${ }^{1}$ publicaron su modelo epidemiológico en el que establecían los diversos estadios que atraviesa una población cuando se introduce el consumo de tabaco. Este modelo ha resultado de mucho interés al poner de manifiesto, entre otras cosas, cómo se extiende la epidemia del consumo

(I) Unidad de Tabaquismo de la Universidad de Zaragoza. Departamento de Medicina y Psiquiatría de la Universidad de Zaragoza. Zaragoza, España.

(2) Programa de salud maternoinfantil. Dirección General de Salud Pública. Departament de Salut. Generalitat de Catalunya. Cataluña, España.

Fecha de recibido: 26 de marzo de 2010 - Fecha de aceptado: 4 de junio de 2010

Solicitud de sobretiros: Dra. Isabel Nerín. Departamento de Medicina y Psiquiatría, Facultad de Medicina. Edificio B, C/Domingo Miral s/n. 50009 Zaragoza, España. Correo electrónico: isabelne@unizar.es 
de tabaco en una población según el género y las consecuencias que tiene sobre la salud en ambos sexos.

Sexo y género no son sinóminos, aunque a veces en la literatura anglosajona pueden sustituir una palabra por otra. ${ }^{2} \mathrm{El}$ concepto de género es una construcción cultural en la que influye el contexto social de cada momento, mientras que el sexo está determinado biológicamente: se nace con un sexo; el género se hace, se construye socialmente. El género actúa como principio organizador de la estructura social posibilitando el acceso a recursos sanitarios y la distribución de los mismos. Por otra parte, el género subyace en la base de los valores y normas de las estructuras sanitarias asistenciales, investigadoras, académicas, planificadoras y preventivas. ${ }^{3}$

Diferenciar las estadísticas por sexos ya ha sido una forma de saber qué es lo que les ocurre a mujeres y hombres en determinadas enfermedades o en relación con condiciones concretas de vida o trabajo; ha sido un primer paso para visibilizar las diferencias. ${ }^{2}$ Pero este análisis de resultados no siempre se ha hecho teniendo en cuenta la perspectiva de género, es decir, entendiendo que los roles de género atribuidos por la sociedad a cada uno de los sexos condicionan diferencias tanto en la manera de enfermar o sanar como en el acceso al sistema y la oferta de recursos sanitarios.

El consumo de tabaco en la mujer ha tenido un claro componente de género sin el que es difícil comprender el pasado reciente y el futuro inmediato. ${ }^{4}$ La mujer se inicia en el consumo de tabaco más tarde que el hombre, condicionada por factores socioculturales relacionados con su incorporación al mundo laboral y el movimiento de igualdad de derechos. Esta influencia que tiene (y ha tenido) el contexto sociocultural justifica la marcada diferencia que existe en la prevalencia de tabaquismo entre las mujeres de países en desarrollo (9\%) y las de países desarrollados (15-22\%), en alguno de los cuales es superior a la de los varones. ${ }^{5}$

El modelo epidemiológico de López y colaboradores muestra también cómo las consecuencias sobre la salud por fumar cigarrillos se producen más tarde en las mujeres que en los varones, debido a la diferente cronología en el consumo. ${ }^{1}$ Estas diferencias de género han condicionado que las enfermedades producidas por el tabaco hasta hace poco tiempo hayan sido mayoritariamente "cosa de hombres", lo que ha contribuido a que todavía hoy exista una baja sensibilización como problema de salud pública específico para la mujer. ${ }^{4}$

Dada la creciente prevalencia de tabaquismo entre las mujeres, es necesario, por tanto, incorporar la perspectiva de género no sólo en el análisis del problema, sino también en el diseño de políticas cuyo objetivo final sea la prevención y el tratamiento del tabaquismo en este grupo de riesgo que en la actualidad son las mujeres de cualquier entorno y de cualquier país, adaptadas a la fase de la epidemia tabáquica en que se encuentren.

Este artículo pretende revisar las estrategias más efectivas para reducir el tabaquismo en los distintos grupos de mujeres que integran la población femenina en su conjunto y plantear posibles retos que todavía hoy nos presenta el control de la epidemia tabáquica actual.

\section{Marco global de control del tabaquismo}

La Organización Mundial de la Salud (OMS) prevé que la prevalencia de tabaquismo entre las mujeres de todo el mundo será de $20 \%$ en el año 2025, en contraste con el $12 \%$ actual. ${ }^{6}$ El tabaquismo femenino está reduciéndose en países como Reino Unido, Estados Unidos, Australia y Canadá, mientras que en algunos países del este y sur de Europa la prevalencia es estable o sigue aumentando. En la región de las Américas las prevalencias pueden ir desde 5.6\% como en Ecuador, hasta 30.5\% como en Chile, por citar algún ejemplo. ${ }^{5}$

Dada la gran variabilidad existente entre los países y la tendencia al aumento de las prevalencias entre las mujeres, es necesario un marco global de control del tabaco que permita la protección de los derechos constitucionales a la salud, a la información, al disfrute de un medio ambiente más saludable, tanto de las personas que fuman como de quienes nunca han fumado o han dejado de fumar, así como a medidas de prevención y asistencia sensibles a los aspectos de género.

Por otra parte, para que las distintas intervenciones de prevención y asistencia en relación con el tabaquismo desarrolladas desde distintos sectores vean incrementada su efectividad, es esencial la existencia de un marco político global de control del tabaco y de los factores que promueven su consumo.

Es interesante observar la evolución de las políticas a lo largo del tiempo según el clima social existente y la evidencia sobre los efectos del tabaco en la salud (cuadro I). ${ }^{7}$ Dado que también cambia la población fumadora, estas políticas deben adaptarse a las necesidades de las diferentes poblaciones y, por lo tanto, incorporar la perspectiva de género y contemplar otras desigualdades sociales, aspectos ambos que constituyen un reto todavía pendiente para la mayoría de los países.

En relación con el género, se han descrito tres clases de políticas: las neutrales, que asumen que influyen de la misma manera en ambos sexos, es decir, que no contemplan que hombres y mujeres se comportan de manera diferente; las sensibles, que incluyen la perspectiva de género y por último las redistributivas cuyo objetivo es establecer una relación más equilibrada entre hombres y mujeres. ${ }^{8}$ Como se observa en el cuadro I, hasta ahora 
Cuadro I

EVOLUCIÓN HISTÓRICA DE ASPECTOS SOCIALES RELACIONADOS CON LA EPIDEMIA TABÁQUICA

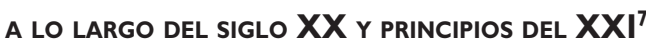

Población fumadora

Hombres

Hombres/inicio en las mujeres

Hombres y mujeres

Hombres y mujeres

Mujeres $y$ hombres

Poblaciones desfavorecidas

ambos sexos

*Deben incluir la perspectiva de género y contemplar otras desigualdades
Clima social

Políticas de control y prevención

Evidencia de los efectos sobre la salud las políticas de control del tabaquismo se pueden clasificar como neutrales en relación con el género. Debido a que los distintos países se encuentran en diferentes fases de la epidemia no es posible establecer un marco temporal único. En aquellos países en los que primero se extendió el consumo de tabaco (países anglosajones) el esquema se ajusta desde principios del siglo XX hasta la actualidad, con tres periodos temporales: primera mitad del siglo $X X$, segunda mitad del siglo XX y el inicio del siglo XXI.

Las políticas de control del tabaquismo deben cubrir tres objetivos principales: la prevención del inicio del consumo de tabaco, la asistencia para aquellas personas que desean dejar de fumar y la protección de toda la población frente al humo ambiental del tabaco (HAT), incluyendo con especial énfasis a la población más joven y las mujeres embarazadas.

Para garantizar este enfoque integral, las políticas dirigidas al control del tabaquismo deben incorporar los aspectos específicos de género y tener en cuenta las especificidades de cada grupo de edad, colectivo o procedencia tal como recomienda el Convenio Marco para el Control del Tabaco (CMCT) de la Organización Mundial de la Salud (OMS). ${ }^{9}$

\section{La perspectiva de género en las políticas de control del tabaquismo}

Un aspecto relevante para el control del tabaquismo en la actualidad es el conocimiento de que en las fases más avanzadas del modelo epidemiológico aparecen grandes desigualdades socioeconómicas y de género, que condicionan el consumo de tabaco entre los sectores mas desfavorecidos. ${ }^{10}$ Por tanto, cualquier medida de control del tabaquismo debería tener en cuenta estas desigualdades, en general, y la desigualdad de género, en particular.

Un informe del Foro Económico Mundial ${ }^{11}$ que incluye la salud y el bienestar como indicadores clave de la igualdad, concluye que, aunque ningún país ha conseguido la igualdad, algunos países presentan diferencias de género considerablemente menores que otros. Según este informe, los países escandinavos son los primeros en igualdad de género, seguidos de Nueva Zelanda, Canadá, Reino Unido, Alemania y Australia. Estados Unidos aparece en el puesto 17, mientras que España está en el lugar 27. Respecto de Latinoamérica, el país más igualitario es Costa Rica (puesto 18), seguido por Colombia (30), Uruguay (32) y Argentina (35). Más abajo en la lista aparecen Perú (47), Chile (48), Venezuela (49), Brasil (51) y México (52).

Existe la peligrosa y extendida percepción de que el tabaco es símbolo de igualdad e independencia, mensaje que la industria del tabaco, conocedora de la importancia que tienen estos valores, ha utilizado sistemáticamente en las campañas publicitarias destinadas a incrementar el consumo de tabaco entre las mujeres.

Es evidente que las consecuencias de fumar para las mujeres no mejoran las condiciones de desigualdad, sino más bien al contrario: empeoran su salud y por tanto ponen en peligro sus perspectivas de igualdad económica, sanitaria y de género. Por el contrario, al mejorar la salud de las mujeres se les capacita para mejorar su estatus y, por tanto, su propia capacidad de decisión y autonomía, además de que contribuye a disminuir las desigualdades.

Determinadas estrategias utilizadas en el control del tabaquismo pueden reforzar la desigualdad de género 
existente entre ambos sexos, mientras que otras pueden transformarla a la vez que logran reducir el consumo. Por ejemplo, los mensajes simplistas que vinculan el consumo de tabaco a la liberación de las mujeres, el uso de medidas paternalistas protectoras sobre las mujeres o focalizar la reducción del consumo sólo durante el embarazo y el cuidado de los niños pequeños, refuerzan el papel tradicional de las mujeres y no suponen, por tanto, medidas adecuadas. Por el contrario, aquellas políticas de control del tabaquismo que promueven un análisis crítico y buscan corregir las desigualdades entre hombres y mujeres, junto con la reducción del consumo de tabaco, contribuyen a transformar la desigualdad y por ello son las que se deben recomendar. ${ }^{12}$

Por otra parte, la experiencia de diversos países muestra que a pesar de la eficacia de las diversas políticas de control desarrolladas, en la actualidad son necesarias medidas de sensibilización de género para influir en el consumo de tabaco.

A continuación se comentan las diferentes políticas de control del tabaquismo con las estrategias mínimas que deberían incluir, incorporando los aspectos específicos de género (cuadro II). Se describen, en primer lugar, las medidas de empoderamiento destinadas, en principio, a disminuir la desigualdad de género, pero que por razones obvias tienen su indicación en el problema del tabaquismo en las mujeres.

\section{Cuadro II}

Políticas del control del tabaQuismo

\begin{tabular}{|c|c|}
\hline Líneas politicas & Estrategias \\
\hline A. Empoderamiento & $\begin{array}{l}\text { - Medidas educacionales } \\
\text { - Prevención en la escuela } \\
\text { - Campañas mediáticas } \\
\text { - Movimientos asociativos femeninos }\end{array}$ \\
\hline B. Legislativas & $\begin{array}{l}\text { - Protección del derecho a la salud de las mujeres } \\
\text { (ambientes libres de humo) } \\
\text { - Control del producto, de su accesibilidad y dis- } \\
\text { - Menibilidad } \\
\text { - Medidas fiscales }\end{array}$ \\
\hline $\begin{array}{l}\text { C. Medidas } \\
\text { asistenciales }\end{array}$ & $\begin{array}{l}\text { - Tratamiento de deshabituación con incorporación } \\
\text { de otros aspectos (control del peso, del estrés...) } \\
\text { - Evitar sesgos de género en la oferta asistencial }\end{array}$ \\
\hline $\begin{array}{l}\text { D. Investigación } \\
\text { y evaluación }\end{array}$ & $\begin{array}{l}\text { - Incorporación de indicadores sensibles a las des- } \\
\text { igualdades de género en las encuestas de salud } \\
\text { rutinarias (monitorización) } \\
\text { - Evaluación de los programas teniendo en cuenta } \\
\text { las desigualdades de género }\end{array}$ \\
\hline
\end{tabular}

\section{Medidas de empoderamiento que permitan a las mujeres obtener un mayor control sobre el tabaquismo}

El término de empoderamiento (del inglés empowerment) fue adoptado en 1995 en la Coferencia Mundial de la Mujer celebrada en Pekín, para referirse al aumento de la participación de las mujeres en los procesos de toma de decisiones, incluidos los que les atañen a las propias mujeres de manera individual. Se trata de un proceso por el cual las mujeres fortalecen sus capacidades, confianza, visión y protagonismo como grupo social para impulsar cambios positivos de las situaciones que viven; incluye tanto el cambio individual (como mujer) como la acción colectiva (como grupo de mujeres).

Dentro de este apartado se pueden contemplar, además de la educación, los programas de prevención en la escuela, las campañas informativas y el movimiento asociativo femenino.

\section{Medidas educacionales}

El empoderamiento educacional de las mujeres puede contribuir a reducir el consumo de tabaco. ${ }^{12}$ La importancia de la educación, formación y conciencia pública sobre los peligros de los productos del tabaco, así como sobre los engaños para captar consumidoras de la industria tabaquera, son un aspecto esencial de las actividades de control del tabaco en las que pueden estar al frente los gobiernos y la sociedad civil. Resulta vital que las mujeres estén implicadas directamente en preparar esta información, incluida la contrapublicidad, especialmente cuando ésta se dirige a mujeres y chicas. ${ }^{13}$ Estos esfuerzos educacionales pueden requerir programas a medida, puesto que la mayoría de la población que no tiene acceso a una educación básica esta compuesta por mujeres.

\section{Los programas de prevención escolar}

Los programas de prevención del consumo de tabaco en la escuela en principio están destinados a adolescentes de ambos sexos. La adolescencia es la etapa de la vida donde se construye la propia identidad en la que intervienen e interactúan diversos factores. Por ello, en lo que concierne a las niñas y a las adolescentes, estos programas se pueden considerar como un instrumento útil para capacitarlas (empowerment) para la propia toma de decisión en relación con el inicio en el consumo de tabaco.

Dichos programas retrasan la iniciación al tabaquismo, y su evaluación a corto plazo presenta resultados positivos. A más largo plazo, los resultados presentan 
inconsistencias y la evaluación ha sido menor. Es especialmente menos conocido lo que sucede cuando se pasa de un lugar altamente controlado en investigación y los programas se aplican en escuelas a gran escala. ${ }^{14}$ Los programas que mejores resultados han dado son aquellos basados en el modelo de influencia social, que incluyen sesiones de refuerzo, que se realizan de una forma interactiva y que se llevan a cabo por los iguales ("peers"). ${ }^{15}$

Hasta el momento no se ha realizado un esfuerzo sistemático para desarrollar y evaluar intervenciones preventivas específicas por género, a pesar de que la prevalencia de tabaquismo entre las jóvenes está superando a la de los chicos en determinados países, incluidos algunos de Latinoamérica. ${ }^{13}$ Algunos autores anglosajones postulan que la falta de efectividad de los programas de prevención tabáquica en el ámbito escolar es, en parte, debida a la falta de especificidad y sensibilidad de género de los mismos. ${ }^{16}$ Dichos programas no incidirían en algunos aspectos de género más predominantes en el tabaquismo femenino como es el caso de la autoestima o la imagen corporal.

En el ámbito educativo también es recomendable utilizar las sinergias de distintas intervenciones en la comunidad y en la escuela ${ }^{17}$ como, por ejemplo, la incorporación de aspectos educativos en las asociaciones de padres de alumnos y, por supuesto, la creación de políticas libres de humo en los centros docentes que facilitarían y apoyarían todos los esfuerzos dirigidos a las jóvenes en el campo que nos ocupa. Asimismo, las intervenciones educativas y de ayuda a la cesación, dirigidas al colectivo docente que juega un importante rol de modelo social y que presenta prevalencias de tabaquismo elevadas, tendrían también efectos beneficiosos.

\section{Campañas informativas de sensibilización sobre el tabaquismo y las estrategias de promoción encubierta}

Las campañas informativas mediáticas, siempre y cuando se diseñen de forma planificada, se implementen de manera sostenida y conjuntamente con otras estrategias, pueden presentar resultados positivos. En efecto, las campañas tienen la capacidad de poder difundirse en segmentos amplios de la población, llegando incluso a los grupos que presentan niveles educativos más desfavorecidos, pudiendo disminuir los obstáculos que tienen estas personas para poder asistir a los programas relacionados con la salud..$^{18}$ Las intervenciones mediáticas, para que sean más efectivas, han de basarse en técnicas de "marketing social", incorporando enfoques estructurados para influir en las actitudes y en los comportamientos relacionados con la salud pública. ${ }^{14}$
Existen medios de comunicación cuyo público mayoritario son las mujeres y que podrían utilizarse para difundir campañas sensibles a los aspectos específicos de género femenino; máxime cuando muchos de estos medios, como se ha podido analizar en algunos estudios cualitativos, son utilizados por la industria tabaquera para hacer promoción encubierta de los productos del tabaco, aun cuando la promoción y la publicidad directa estén prohibidas. En efecto, la industria tabaquera analiza las razones por las cuales las mujeres fuman, observa las diferencias en grupos de edad concretos (pe: las mujeres jóvenes) para después representarlas gráficamente en la iconografía de las revistas. Los resultados de estas intervenciones han sido beneficiosos para la industria tabaquera y han mostrado la vulnerabilidad de las jóvenes a la publicidad de promoción del tabaco. ${ }^{19}$ Para contrarrestar este efecto, es esencial difundir la idea que la mujer ideal es aquella que no fuma en todos los ámbitos, intentando reducir la percepción subjetiva errónea que exagera la prevalencia real del consumo. ${ }^{20}$

Actualmente, sería recomendable dirigir las campañas mediáticas hacia los grupos femeninos más desfavorecidos socioeconómicamente, en aquellos países en los que estos grupos se están incorporando al consumo, por resultar más costoefectivas. ${ }^{18}$ En cualquier caso, las campañas de información y sensibilización deben mantenerse en el tiempo, adaptarse a la sensibilidad, al lenguaje, al nivel cultural del público a quien van dirigidas $\mathrm{y}$, por último, complementarse con el desarrollo mantenido de programas educativos en el ámbito escolar, tal como ya hemos comentado, y con el apoyo a la participación de la sociedad civil en el desarrollo de una política coordinada de prevención y control del tabaquismo de las mujeres. ${ }^{21}$

\section{Promoción y apoyo de la prevención del tabaquismo en el movimiento asociativo femenino}

A pesar de que durante años el movimiento de control del tabaquismo no ha tenido mucho éxito en involucrar a las organizaciones femeninas en el control del tabaquismo femenino, es esencial su incorporación en este tema, no sólo porque representan un grupo considerable de mujeres que facilitaría la difusión de los mensajes, sino por la capacidad de influencia y credibilidad que poseen entre sus miembros. ${ }^{18}$

Las funciones que estas organizaciones pueden ejercer se encuentran resumidas en el cuadro III.

Además, estas funciones pueden plantear actuaciones que provoquen interés social sobre la problemática del tabaquismo y muevan a sus miembros a llevar a cabo acciones positivas y estar alerta de cualquier estrategia 


\section{Cuadro III}

FUNCIONES DEL MOVIMIENTO ASOCIATIVO 22

- Sensibilización, educación y movilización

- Identificación y denuncia de promoción encubierta

- Desarrollo de liderazgo por parte de las mujeres

promocional de la industria tabaquera, especialmente de sus formas más sutiles dirigidas a las niñas, adolescentes y jóvenes. En cuanto al desarrollo de liderazgo por parte de las mujeres, este es uno de los aspectos principales y fue lo que motivó la creación de una red femenina de control del tabaquismo como es la International Network of Women Against Tobacco (INWAT). ${ }^{12}$ Esta red surgió como deseo de comunicar y crear una red de control del tabaquismo a nivel internacional en el ámbito de las mujeres. Siguiendo en la misma línea, se han creado otros grupos como, por citar un ejemplo, el grupo de Género y Tabaco del Comité Nacional de Prevención del Tabaquismo en España que aglutina a diferentes profesionales que trabajan en tabaquismo. ${ }^{7}$

La inclusión de organizaciones no gubernamentales y la creación de redes globales de mujeres que trabajen en el desarrollo de políticas de control del tabaquismo son fundamentales para situar de manera equitativa las necesidades de las mujeres en la agenda internacional. ${ }^{12}$

\section{Medidas legislativas}

A lo largo de los años, en la mayoría de los países se ha ido desarrollando un marco legal regulador sobre los diferentes aspectos relativos a la comercialización, promoción y consumo de tabaco. Este proceso se vio reforzado por la puesta en marcha del Convenio Marco de Control del Tabaquismo, que establece las bases para la implementación de medidas universales en este ámbito y que defiende la importancia del protagonismo de las mujeres en el tema del tabaco. ${ }^{23}$

En España, la reciente Ley 28/2005 “de medidas sanitarias frente al tabaquismo y reguladora de la venta, el suministro, el consumo y la publicidad de los productos del tabaco", ${ }^{24}$ que entró en vigor en enero de 2006, es la primera ley relacionada con el control del tabaquismo que contempla y señala explícitamente la necesidad de incorporar la perspectiva de género en todas las estrategias que se lleven a cabo para controlar el tabaquismo y reconoce que "el fenómeno del tabaquismo no se manifiesta de igual manera en hombres y mujeres...." Se trata además de una ley que cuenta con un elevado grado de soporte social, y en este sentido hay que destacar que las mujeres españolas parecen más receptivas y sensibles a la necesidad de su entrada en vigor y aplicación. Así, según una encuesta reciente realizada por el Comité Nacional para la Prevención del Tabaquismo (CNPT), ${ }^{*} 70.3 \%$ de las mujeres contra $66.5 \%$ de los hombres estarían más de acuerdo con la nueva legislación.

A continuación se enumeran las medidas legislativas relacionadas con la protección del derecho a la salud (ambientes libres de humo) y las relacionadas con el control del producto, su accesibilidad y su disponibilidad.

\section{Protección del derecho a la salud de las mujeres}

Dado que existe evidencia suficiente sobre los efectos perjudiciales sobre la salud de la exposición al humo ambiental del tabaco (HAT), ${ }^{25}$ se hace necesario que todos los lugares públicos cerrados, y en especial todos los centros de trabajo, sean espacios realmente libres de humo, no sólo para proteger la salud de las personas que no fuman, sino también para difundir el mensaje social de la incompatibilidad entre consumo de tabaco y salud, así como para facilitar la cesación tabáquica. ${ }^{26}$

Un sector relevante en el caso de las mujeres activas laboralmente es la hostelería, sector que ofrece más oportunidades de trabajo para las mujeres, incluso a las que tienen escasa preparación y deficiencias en el manejo de un idioma extranjero, por lo que es habitual que muchas inmigrantes accedan a estos puestos de trabajo. ${ }^{27}$ De hecho, es un sector en el que suele trabajar un mayor porcentaje de mujeres que de hombres, como por ejemplo en el caso de España, donde 53\% de las personas trabajadoras en la hostelería son mujeres. ${ }^{28}$

Estas políticas son sobre todo efectivas para reducir la prevalencia del tabaquismo y también para disminuir el consumo diario de cigarrillos. ${ }^{29}$ En cuanto al efecto diferencial de género parece que los varones disminuyen su consumo de forma mayoritaria que las mujeres, aunque todavía se requeriría una mayor investigación del problema, pues aparecen resultados divergentes según el lugar público analizado. ${ }^{30}$

Aunque los potenciales beneficios de los ambientes libres de humo son evidentes, la presencia o ausencia de estas medidas y su cumplimiento dependen de la región geográfica, de la ocupación y de la actividad

\footnotetext{
* Encuesta sobre conocimientos, actitudes, creencias y conductas en relación al consumo de tabaco. Comité Nacional de Prevención del Tabaquismo, diciembre 2006 (inédito).
} 
industrial, entre otros factores. La creación de espacios libres de humo, tanto laborales como domésticos, puede ser potencialmente más difícil de alcanzar para aquellas mujeres y chicas de niveles socioeconómicos más desfavorecidos. En ambos entornos, tanto el doméstico como el laboral, influye además la desigualdad de género, puesto que el reparto desigual del poder hace que la capacidad de estas mujeres para controlar el ambiente en el que viven o trabajan sea limitada, ${ }^{31}$ de ahí la necesidad de las medidas de empoderamiento ya comentadas en otro apartado y la necesidad de adaptarlas al nivel cultural y socieconómico de las diferentes poblaciones de mujeres. En este sentido, resulta de interés no sólo proporcionarles información sobre los efectos perjudiciales sobre su salud, sino también que sepan cómo funciona la legislación laboral en su país y cómo utilizarla para protegerse en ese ambiente, de la misma manera que puede ser útil trasladar esta información a las organizaciones que trabajan con y para las mujeres. ${ }^{13}$ Por otra parte, y respecto al entorno doméstico, es menos probable que los adolescentes que viven en hogares libres de humo experimenten con los cigarrillos, pues se muestra que los hogares más permisivos tienen una mayor influencia en las chicas para incorporarse al consumo de tabaco. ${ }^{32}$

Para que las políticas dirigidas a la creación de lugares públicos y de trabajo libres de humo tengan éxito es necesario, por no decir imprescindible, que además de implementarse sobre poblaciones altamente informadas sobre los efectos secundarios del la exposición al humo ambiental del tabaco, éstas posean un alto grado de apoyo y de aceptabilidad social. ${ }^{33}$

\section{Control del producto, de su accesibilidad y disponibilidad}

La publicidad, la promoción y el patrocinio del tabaco y otros productos relacionados, han sido y son algunos de los factores que más han incidido en la expansión y el mantenimiento del tabaquismo en todo el mundo. Su prohibición es un aspecto decisivo en cualquier política de prevención y control del tabaquismo, pues las estrategias de promoción han contribuido de forma sensible a que la epidemia del tabaquismo se siga manteniendo a pesar del conocimiento actual del problema, a pesar del incremento de la mortalidad entre la población fumadora, y a pesar del incremento constante de la proporción de personas que han dejado de fumar a lo largo de las últimas décadas. Lo anterior, junto con la accesibilidad y la disponibilidad del producto, son parte de las estrategias comerciales y ambientales que condicionan el inicio del consumo de tabaco y sobre las cuales han actuado hasta ahora las políticas de control de tabaquismo.
Control de las estrategias de promoción

Para que la prohibición de la promoción del tabaco sea efectiva debe ser total, pues las restricciones parciales obtienen un impacto reducido al permitir a las compañías tabacaleras la utilización de distintas estrategias promocionales, especialmente dirigidas a la población más joven y a las mujeres.

Existe suficiente evidencia sobre el efecto disuasor del consumo imputable a la supresión total de la publicidad de tabaco. En los países donde se ha aplicado una ley de prohibición total de publicidad de tabaco se ha experimentado un descenso significativo del consumo ${ }^{34}$ y esta disminución significativa se ha mantenido a largo plazo. ${ }^{35}$ Los datos aportados por Noruega, Finlandia, Nueva Zelanda y Francia apuntan a que -incluso en distintos contextos culturales- la prohibición total de la publicidad, cuando va acompañada de una política coherente de control del tabaquismo, ha conseguido disminuir a largo plazo entre 10 y $37 \%$ el consumo per cápita de tabaco en estos países. ${ }^{35-38}$

Las estrategias de publicidad y promoción han centrado sus esfuerzos en los grupos de población más vulnerables, como son las mujeres, y en especial aquellas procedentes de los grupos sociales más desfavorecidos. La publicidad muestra el cigarrillo como símbolo de igualdad, de liberación y poder para las mujeres jóvenes y adolescentes, de triunfo en el mundo laboral, de placer, de capacidad de seducción y éxito en la vida, así como un eficaz medio para combatir el estrés. Respecto a las mujeres de los grupos sociales más desfavorecidos, se presenta al cigarrillo como símbolo de alto estatus económico, de éxito en la vida y de modernidad.

Otro aspecto de la publicidad del producto de tabaco está relacionado con la prohibición de las palabras "mild" o "light". Dicha prohibición beneficia fundamentalmente a las mujeres que siempre se han mostrado más sensibles a los aspectos de protección de la salud y por tanto eran más susceptibles al mensaje engañoso de que los cigarrillos "light" son menos nocivos para su salud. Sin embargo, a pesar de la prohibición la industria tabacalera continúa intentando diferenciar el tabaco "light" del "normal" mediante la utilización de diseños y colores evocadores de entornos "limpios" y paradisíacos. Por ello, se debería avanzar hacia la introducción de la comercialización de cajetillas genéricas, con el mismo color de fondo para todas las marcas y tipos de cigarrillos, de cara a proteger a las mujeres de este consumo. ${ }^{21}$ En relación con el etiquetado y empaquetado, las advertencias sanitarias que incluyen mensajes en forma de imágenes pueden tener un gran impacto, especialmente en países en desarrollo donde los niveles de analfabetismo entre las mujeres son altos. ${ }^{13}$ 
Disminución de la accesibilidad de los productos del tabaco

La accesibilidad a los productos derivados del tabaco es un factor ambiental nada despreciable que influye en la iniciación en el tabaquismo, especialmente durante la adolescencia. A pesar de que en muchos países existe una legislación que prohíbe la venta de tabaco a menores de edad, ésta se sigue produciendo ya sea directamente o a través de un acceso fácil al mismo, como por ejemplo, vía las máquinas expendedoras automáticas. Además, ciertas problemáticas como la venta de cigarrillos sueltos en el entorno escolar o la aparición de marcas que ofrecen paquetes de 10 unidades de cigarrillos, cuyo precio resulta asequible para los y las jóvenes, son difíciles de controlar. ${ }^{21}$

Diversos estudios anglosajones han puesto de manifiesto la existencia de una mayor facilidad por parte de los comerciantes para vender cigarrillos a las chicas que a los chicos de la misma edad, ${ }^{22}$ aunque parece ser que las chicas utilizan de forma más frecuente que los chicos otras vías no comerciales de obtención de cigarrillos. ${ }^{39}$ Asimismo, la evaluación del impacto de la aplicación de la ley, junto con otras intervenciones como la educación de la comunidad, ha mostrado una reducción significativa de las ventas de tabaco a menores de edad de ambos sexos. Sin embargo, estos mismos estudios concluyen que la evidencia objetiva de que este cumplimiento se traduzca en una reducción del consumo por parte de los y las jóvenes sigue siendo limitada. En efecto, la juventud utiliza distintos medios para obtener cigarrillos -a través de familiares, amigos y extraños- que son mucho más difíciles de controlar. ${ }^{14}$

Disminución de la disponibilidad: políticas fiscales y de precios

El incremento de los precios de cigarrillos no sólo previene el inicio del tabaquismo y favorece la cesación por parte de aquellas personas que fuman, sino que también tiene un efecto beneficioso en la reducción de las recaídas en aquellas personas que han dejado de fumar.

La elasticidad de la demanda es mayor en los países en vías de desarrollo, y por ello las políticas fiscales tienen también más impacto en los grupos de menor renta de los países ricos. En países como el Reino Unido o Suecia, donde se han llevado a cabo incrementos importantes de los impuestos sobre el tabaco, se ha demostrado una reducción significativa de su consumo directamente relacionada con el incremento de los precios. ${ }^{40,41}$

El efecto del incremento de los precios e impuestos del tabaco depende del sexo, del nivel socioeconómico y de la edad del grupo poblacional al que afecta la medida. El grupo más sensible al aumento de precios es el de los y las jóvenes puesto que llevan menos tiempo fumando y pueden adaptarse mejor a los cambios, tienen menor poder adquisitivo y a ellos o ellas les afecta más la conducta de los iguales ("peers"). ${ }^{14}$ Sin embargo, a juzgar por los resultados de diversos estudios realizados al respecto, parecería ser que los chicos fueran más susceptibles a las políticas de incrementos de precios del tabaco, presentando una mayor elasticidad de la demanda que las chicas. En concreto, un estudio anglosajón ha estimado una reducción de la demanda de $9.2 \%$ en el caso masculino, frente a una disminución de la misma en las chicas de $5.9 \%$, ante $10 \%$ de aumento del precio del tabaco. ${ }^{31}$

En el caso de las mujeres, y en general de los grupos económicos de menor poder adquisitivo, el incremento de impuestos puede plantear problemas éticos. Existe todavía un debate abierto para contestar a la pregunta de si el aumento de los impuestos del tabaco podría ser regresivo o progresivo en estos grupos desfavorecidos. ${ }^{42,43}$ Lo que sí es evidente es que en aquellos países en los cuales las prevalencias más elevadas de tabaquismo se centran en los grupos más desfavorecidos es donde el aumento de precios tendrá una mayor carga, justamente en aquellas personas que ya tienen dificultades en "llegar a fin de mes".

Por ello, una política al alza de los precios del tabaco mediante impuestos, imprescindible en los países en los que todavía existe un margen importante para aumentarlos, debería ir acompañada de una estrategia basada en la reducción del daño que ocasiona el tabaco, mediante la oferta de terapias eficaces para que las fumadoras de mayor riesgo pertenecientes a los grupos más desfavorecidos consigan dejar de fumar y consolidar la abstinencia. ${ }^{7}$

\section{Intervenciones de ayuda para dejar de fumar sensibles a las necesidades de género}

En la actualidad no existe un acuerdo acerca de si hay diferencias según sexo en los resultados del tratamiento del tabaquismo. En este sentido, tampoco hay muchos estudios que hayan realizado análisis por sexo y que contemplen variables relacionadas con el género, por lo que se hace necesario realizar más investigación en este tema.

La principal barrera a la cesación tabáquica para hombres y mujeres es la adicción a la nicotina, pero en el caso de las mujeres parece que existen otros factores que deberían formar parte de estos programas de forma que, cuando fuera necesario, además del tratamiento farmacológico se pudiera hacer énfasis en aspectos como 
el control del peso, el incremento de ejercicio físico, las estrategias de manejo del estrés, o bien, las intervenciones de gestión del propio estilo de vida. ${ }^{44}$ También sería recomendable la monitorización de la presencia de síntomas depresivos antes, durante y después del tratamiento indicado para abandonar el tabaquismo. ${ }^{11}$ Por otra parte, es interesante recordar que las mujeres suelen tener más facilidad para enrolarse en cualquier programa formal de ayuda a la cesación tabáquica. ${ }^{*}$

En la atención sanitaria cabe recordar el sesgo de género que ha existido hasta ahora basado en la consideración de las mujeres como consumidoras de menor riesgo, lo que ha condicionado una percepción errónea tanto en las propias mujeres como entre los y las profesionales que han incidido en menor grado en la ayuda que requería este grupo. ${ }^{4}$ Es importante que los y las profesionales de la salud cuenten con la información necesaria para que tengan en cuenta esta situación, ya desde la etapa de pregrado, ${ }^{45}$ y evitar este sesgo de género ya descrito en la cardiopatía isquémica y en la enfermedad pulmonar obstructiva crónica.

Existen distintos niveles de asistencia donde se puede intervenir y ayudar a las mujeres. Desde el consejo médico de baja intensidad pero de gran alcance ("intervención mínima"), realizado en atención primaria y también en atención especializada por parte de profesionales de otros ámbitos como de salud sexual y reproductiva y de pediatría, hasta una intervención más intensiva desde los servicios de atención especializada de tabaquismo. ${ }^{46,47}$

Además de las actuaciones terapéuticas en contextos asistenciales tradicionales, en el caso de las mujeres es particularmente importante incorporar otro tipo de programas comunitarios de apoyo para dejar de fumar. Dichos programas se basan en el reconocimiento de la importancia de los componentes sociales de la dependencia y de la motivación, de gran peso en el colectivo femenino; se trata de programas que alcanzan a toda la población y que pueden lograr tasas relevantes de abstinencia a bajo costo. ${ }^{21}$

\section{Investigación y evaluación: la incorporación de indicadores sensibles a las diferencias de género}

No podemos olvidar que para conocer lo que está pasando, entender la evolución del problema y, sobre todo, saber si las medidas adoptadas han sido efectivas, es esencial un sistema de información y registro que abar-

* Department of Health. Statistics on smoking cessation services in health authorities. London: Department of Health, 2000 (inédito). que indicadores específicos de género, es decir, que no sólo nos permita estratificar por sexo sino que permita conocer más en profundidad otros aspectos como el nivel socioeconómico, el trabajo reproductivo y remunerado, el nivel cultural, las actitudes, los conocimientos, las expectativas, etc., variables que recogen aspectos que condicionan la salud de hombres y mujeres.

Las Encuestas de Salud deben incorporar indicadores específicos de género para mejorar la planificación y su posterior evaluación, ${ }^{48,49}$ y lograr una mejor adecuación de las políticas sociales dirigidas a los colectivos más vulnerables. En este sentido y por citar un ejemplo, el análisis debe permitir reconocer diferencias de género tan claras como que en los hombres el hecho de convivir en pareja representa un factor protector de la salud, mientras que en las mujeres, debido a la distribución desigual del trabajo reproductivo, puede conllevar a un empeoramiento de la salud, que además se ve agravado por el consumo de tabaco y por la imposibilidad de dejarlo.

Asimismo, además de recoger el consumo de tabaco con perspectiva de género en las encuestas nacionales de salud, el indicador de consumo de tabaco debe ser recogido en todas aquellos sistemas de información relacionados con otros ámbitos, como por ejemplo el de la salud perinatal. En definitiva, se requiere un esfuerzo conjunto de vigilancia epidemiológica, a ser posible entre distintos países, que incluya información epidemiológica, económica, determinantes socioeconómicos y de género, de políticas de control y de la industria tabaquera para poder llevar a cabo una buena evaluación del impacto de las estrategias de control del tabaquismo, en este caso concreto entre las mujeres. ${ }^{50}$

\section{Conclusiones}

Las fases más avanzadas de la epidemia tabáquica muestran que las desigualdades sociales, en general, y las de género, en particular, favorecen el consumo de tabaco sobre todo entre las mujeres y los grupos sociales más desfavorecidos. Para modificar esta tendencia del incremento del tabaquismo entre las mujeres resulta prioritario introducir cambios en las políticas de control del tabaquismo desarrolladas hasta ahora.

La construcción social que supone el concepto de género condiciona no sólo el inicio y el mantenimiento del consumo, sino también la oferta de tratamientos eficaces para las mujeres que son fumadoras. La industria tabaquera ha incorporado la perspectiva de género con éxito en sus campañas de promoción destinadas a las mujeres. Es necesario por tanto que desde el campo de la salud pública se incorpore la perspectiva de género tanto en el análisis del problema, con evaluaciones que 
incorporen variables específicas y sensibles a diferencias de género, como en el planteamiento de las posibles soluciones con el diseño de políticas sensibles al género en el control del tabaquismo.

De esta manera se avanzaría enormemente en determinados aspectos como la reducción de las prevalencias en grupos de mujeres jóvenes, embarazadas y de nivel socioeconómico desfavorecido, en el retraso de la edad de inicio del consumo en adolescentes y en un mejor enfoque de género en el tratamiento, y que suponen un reto en las políticas de control del tabaquismo actuales.

\section{Declaración de conflicto de intereses}

Declaramos no tener conflicto de intereses.

\section{Referencias}

I. Lopez A, Collishaw N, Piha T.A descriptive model of the cigarette epidemic in developed countries. Tob Control 1994; 3:242-347. 2.Valls-Llobet C. Invisibilidad de las mujeres para la medicina. En: Estudios Iberoamericanos de género en ciencia, Tecnología y Salud. Miqueo C, Barral Maj, Magallón C (eds). Zaragoza: Prensas Universitarias de Zaragoza,2008:677-683.

3. Ruiz-Cantero T.La perspectiva de género aplicada a la investigación y la asistencia sanitaria. Miqueo C, Barral Maj, Magallón C(eds). Zaragoza: Prensas Universitarias de Zaragoza, 2008:653-664.

4. Nerín I. El tabaquismo en la mujer: una atracción fatal.Arch Bronconeumol 2005; 41: 360-362

5. The Tobacco Atlas. Geneva:World Health Organization, 2004 6. Harlem-Brundtland G. Prólogo. En J.M. Samet, Soon SY(Ed.). Las mujeres y la epidemia del tabaco- Retos para el siglo XXI. Ginebra: Organización Mundial de la Salud, 200I.

7. Nerín I, Jané M (coord). Libro blanco sobre mujeres y tabaco.Abordaje con una perspectiva de género. Zaragoza: Comité Nacional para la Prevención del Tabaquismo y Ministerio de Sanidad y Consumo, 2007. 8. Kabeer N. Gender-aware policy and planning: a social relations perspective. In: Macdonald M, ed. Gender planning in development agencies: meeting the challenge. Oxford: Oxfam, 1994.

9. 56 World Health Assembly WHO Framework Convention on Tobacco control. Document A56-8. Geneva:WHO, 2003.

10. Kunst A, Giskes K, Mackenbach J. EU Network on Interventions to Reduce Socio-economic Inequalities in Health Department of Public Health. Erasmus Medical Center Rotterdam, Netherlands, 2004. [consultado 04/02/20I0]. Disponible en: http://www.ensp.org/files/socio.pdf I I. Lopez-Claros A, Zahidi S. Capacitación de las mujeres: midiendo la brecha de género en el mundo. Ginebra: Foro Económico Mundial, 2005. 12. Greaves L, Tungohan E. Engendering tobacco control: using an international public health treaty to reduce smoking and empower women. Tobacco Control 2007; I6(3): |48-I50.

13. Greaves L, Jategaonkar N, Sánchez S (eds.) Pasando página: Mujeres, tabaco y el futuro. Centro de Excelencia para la Salud de las Mujeres de la Columbia Británica (BCCEWH) y Red Internacional de Mujeres Contra el Tabaco (INWAT).Vancouver: Centro de Excelencia en Salud de las Mujeres de la Columbia Británica, 2006.

14. Lantz PM, Jacobson PD,Warner KE, Wasserman J, Pollack HA, Berson J, et al. Investing in youth tobacco control : a review of smoking prevention and control strategies. Tobacco Control 2000; 9: 47-63
I5. Fernández S, Nebot M, Jané M. Evaluación de la efectividad de los programas escolares de prevención de tabaco, alcohol y cannabis: ¿Qué nos dicen los meta-análisis? Rev Esp Salud Pública 2002; 76: I75-I87. 16. Michell L,Amos A. Girls, pecking order and smoking. Soc Sci Med 1997; 44: 186I-1869

17. Jané-Checa M. Prevención y control del tabaquismo en jóvenes: situación actual y estrategias de futuro. IDEA-Prevención 2002; 24: 78-87 18. Jané M, Pardell H, Saltó E. Prevención del consumo según género. En: Fagerstrom K \& Jiménez C. Tratado de tabaquismo. Madrid:Aula Médica, 2004.

19. Amos A, Gray D, Currie C, Elton R. Healthy or druggy? Self-image, ideal image and smoking behaviour among young people. Soc Sci Med 1997; 45:847-858.

20. Chollat-Traquet C.Women and tobacco. Geneva:WHO, 1992

21. Salvador-Llivinna T. Condicionantes del consumo de tabaco en Espanya. Adicciones 2004; 16:25.

22. US Department of Health and Human Services. Women and Smoking. A Report of the Surgeon General. Rockville, MD: US Department of Health and Human Services, Public Health Service, Office of the Surgeon General, 200I.

23. Nerín I, Jané M (por el Grupo de Trabajo de las Mujeres en Apoyo del Control del Tabaquismo). I Encuentro Nacional de Mujeres en Apoyo al Convenio Marco para el Control del Tabaco. Prev Tab 2003; 5: I5 I-I58 24. Ley $28 / 2005$, de 26 de diciembre, de medidas sanitarias frente al tabaquismo y reguladora de la venta, el suministro, el consumo y la publicidad de los productos del tabaco. BOE n. ${ }^{\circ} 309$, de 27-12-2005: 4224I-50.

25. World Health Organization. International Agency for Reserach on Cancer (IARC). Involuntary Smoking (Group I).Vol 83. Lyon: IARC Monographs, 2002.

26. Jané $M$, Nebot $M$, Rojano $X$, Artazcoz L, Sunyer J, Fernàndez E, et al. Exposure to environmental tobacco smoke in public places in Barcelona, Spain. Tobacco Control 2002; I I:83-84.

27. Moore RS, Lee JP,Antin TMJ, Martin SE. Tobacco free workplace policies and low socioeconomic status female bartenders in San Francisco. J Epidemiol Community Health 2006;60(Suppl II):ii5I-ii56.

28. Instituto Nacional de Estadística. Encuesta de Población Activa 2004. Madrid: INE. [Consulta 6 de junio de 2007]. Disponible en: http:// www.ine.es/inebase/cgi/axi)

29. Farrelly MC, Evans WN, Sfekas AE. The impact of workplace smoking bans: results of a national survey]. Tob Control 1999; 8: 272-277.

30. Chaloupka FJ, Pacula RL. Sex and race differences in young people's responsiveness to price and tobacco control policies. Tobacco Control 1999; 8:373-377.

31. Greaves L,Vallone D,Velicer W. Special effects: tobacco policies and low socioeconomic status girls and women. Journal of Epidemiology and Community Health 2006; 60 (Suppl 2):iil-ii2.

32. Greaves L, Jategaonkar N.Tobacco policies and vulnerable girls and women: toward a framework for gender sensitive policy development. Journal of Epidemiology and Community Health 2006;60(Suppl 2):ii57-65 33. Fernández E,Villalbí JR, Córdoba R.Lecciones aprendidas en el control del tabaquismo en España. Salud Publica Mex 2006;48 supI I:SI48-SI54. 34. Department of Health. Effect of tobacco advertising on tobacco consumption. London: DoH, 1992.

35. Joossens $L$. The effectiveness of banning advertising for tobacco products. Brussels: Ed. International Union Against Cancer, 1997. 36. World Bank. Curbing the epidemic: Governments and the Economics of Tobacco Control.World Bank Development in Practice Series. Washington, DC:World Bank, 1999.

37. Saffer H.The Control of Tobacco Advertising and Promotion. En: Tobacco Control Policies in Developing Countries. Jha P, Chaloupa F, eds. New York : Oxford University Press, 2000.

38. Saffer H,Chaloupka, F.The effect of tobacco advertising bans on tobacco consumption. Journal of Health Economics 2000; I9: I I I7- I I 37. 
39. Castrucci BC, Gerlach KK, Kaufman NJ, Orleans CT.

Adolescents'acquisition of cigarretes through non commercial sources. J

Adolesc Health 2002; 31: 322-326.

40.World Health Organization. Women and the tobacco epidemic.

Challenges for the $2 I^{\text {st }}$ century. Geneva:WHO, 200 I.

41. López-Nicolás A. How important are tobacco prices in the propensity to start and quit smoking? An analysis of smoking histories from the Spanish National Health Survey. Health Econ 2002; I I:52 I-535.

42. Ernster V, Kaufman N, Nichter M, Samet J, Yoon SY.Women and tobacco: moving from policy to action. Bulletin of the World Health Organization 2000; 78: 891-901.

43. Remler DK. Poor smokers, poor quitters, and cigarette tax regressivity. Am J Public Health 2004; 94: 225-229.

44. Jané-Checa M. Mujeres y tabaco:Aspectos principales específicos de género. Adicciones 2004; 16 (supl 2): I-15.

45. Nerin I. Fumando espero. Arch Bronconeumol 2000;36:I I5-I I7.
46. Silagy C, Stead LF. Physician advice for smoking cessation (Cochrane Review). In:The Cochrane Library, Issue 2. Oxford: Update Software, 200 I 47. Greaves L, Cormier R, Devries K, Bottorff J, Johnson J, Kirkland S et al.A best practices review smoking cessation interventions for pregnant and postpartum girls and women.Vancouver: British Columbia Centre of Excellence for Women's Health, 2003.

48. Rohlfs I, Borrell C,Anitua C,Artazcoz L, Colomer C, Escribá V et al. La importancia de la perspectiva de género en las encuestas de salud. Gac Sanit 2000; 14: 146-155.

49. Peiró R, Ramón N, Álvarez-Dardet C, Colomer C, Moya C, Borrell $C$, et al. Sensibilidad de género en la formulación de planes de salud en España: lo que pudo ser y no fue. Gac Sanit 2004; 18: 36-46.

50. Reynales-Shigematsu LM. Costos de atención médica de las enfermedades atribuibles al consumo de tabaco en América: revisión de la literatura. Salud Pública Mex 2006: 48 supl I:190-200. 